\title{
BMJ Open A gender-transformative approach to improve outcomes and equity among persons with traumatic brain injury
}

\author{
Tatyana Mollayeva, ${ }^{\oplus, 2}$ Vanessa Amodio, ${ }^{2}$ Shirin Mollayeva, ${ }^{2}$ Andrea D'Souza, ${ }^{3}$ \\ Heather Colquhoun, ${ }^{1,3}$ Enrico Quilico, ${ }^{3}$ Halina (Lin) Haag, ${ }^{3,4}$ Angela Colantonio ${ }^{1,2,3}$
}

To cite: Mollayeva T, Amodio V, Mollayeva S, et al. A gendertransformative approach to improve outcomes and equity among persons with traumatic brain injury. BMJ Open 2019;9:e024674. doi:10.1136/ bmjopen-2018-024674

- Prepublication history and additional material for this paper are available online. To view these files, please visit the journal online (http://dx.doi. org/10.1136/bmjopen-2018024674).

Received 7 June 2018 Revised 19 February 2019 Accepted 1 April 2019

Check for updates

(c) Author(s) (or their employer(s)) 2019. Re-use permitted under CC BY-NC. No commercial re-use. See rights and permissions. Published by BMJ.

${ }^{1}$ Department of Occupational Science and Occupational Therapy, University of Toronto, Toronto, Ontario, Canada ${ }^{2}$ Research Department, Toronto Rehabilitation InstituteUniversity Health Network, Toronto, Ontario, Canada ${ }^{3}$ Rehabilitation Sciences Institute, University of Toronto, Toronto, Ontario, Canada

${ }^{4}$ Lyle S. Hallman Faculty of Social Work, Wilfrid Laurier University, Ontario, Canada

Correspondence to

Dr Tatyana Mollayeva;

tatyana.mollayeva@utoronto.ca

\section{ABSTRACT}

Introduction The initiation and translation of sex-sensitive and gender-sensitive research programmes into clinically useful considerations for patients with traumatic brain injury (TBI) have been difficult. Clinical frameworks are currently not specific according to sex and gender, despite evidence that these constructs influence the incidence, course and outcome of patients with TBI. The present protocol outlines a strategy for a research programme, supported by the Canadian Institutes of Health Research (CIHR) Institute of Gender and Health, which explores sex and gender topics in the context of TBI, with the goal of building an infrastructure to facilitate the implementation of sex/gender-sensitive research findings into clinical considerations.

Methods and analysis A comprehensive multistep research programme is proposed to support three research objectives: (1) documentation of important concepts and ideas for education on topics of sex and gender in the TBI context using a knowledge-user feedback framework, current scientific evidence and the research team's expertise; (2) development of educational materials for patients with $\mathrm{TBI}$, significant others and clinicians providing care that account for sex/gender and (3) testing the application of these educational materials for feasibility and effectiveness. This programme supports the CIHR Institute's mission by facilitating partnership with knowledge users across clinical, research, academic and community sectors, through a range of platforms and activities.

Ethics and dissemination The Research Ethics Board of the University Health Network has approved the programme. It is anticipated that this work will add significant value to the advancement of the field of sex, gender and health by serving as a model to foster the integration of these constructs across the spectrum of disorders. This will transform clinical practices and ensure that generated knowledge is translated into improved training programmes, policies and health services that are responsive to the diverse needs of men and women with TBI.

PROSPERO registration number CRD42018098697

\section{INTRODUCTION}

The field of sex and gender research is rapidly evolving and growing internationally. ${ }^{1-4}$ The World Health Organization (WHO) has

\section{Strengths and limitations of this study}

- The study of sex and gender in traumatic brain injury (TBI) is important to translate research findings into clinically useful considerations.

- To date, the topic of sex and gender in TBI has not been systematically reviewed, and its value to knowledge users is unknown; this protocol outlines a programme that intends to fill this gap.

- Systematising research data on sex and gender, as well as knowledge users' feedback, is essential for organising educational opportunities, bridging communication between basic science, clinical research and real-life experiences, and encouraging multidisciplinary interactions.

- Platforms that allow knowledge users to interact and share their experiences are basic in this research programme; advanced communication, preferably face-to-face and on a continuum, is needed to support the co-creation process and to ensure value of the product to each knowledge user.

established the term 'gender mainstreaming' to describe the deep-rooted social processes that influence how men and women are treated within society's institutions, tightly associated with the power and privilege that are afforded based on gender. ${ }^{5}$ The WHO has made the recommendation that gender inequality be addressed in all domains, specifically calling for the stratification of data by sex/gender and age in research. ${ }^{5}$ To go about this, it is first important to understand the distinction and the inter-relatedness of the concepts of sex and gender. ${ }^{1-5}$ Sex constitutes the biological and physiological bases that make male, female and intersex individuals distinct from one another; gender, on the other hand, refers to the socially constructed characteristics of people with varying gender identities (ie, norms, behaviours and relations taught based on sex at birth). ${ }^{14}$ Both of these concepts reflect on general health and well-being at multiple levels. ${ }^{3}$ 
The population of interest in the present work is that of persons who have sustained a traumatic brain injury (TBI) ${ }^{6}{ }^{7}$ Definitions of TBI vary; however, all describe structural and/or physiological disruption of brain function as a result of an external force. ${ }^{8}$ Published estimates of TBI are likewise variable, but it is estimated that between 50 and 60 million persons are affected annually worldwide when estimates from studies with comprehensive data collection methods are extrapolated internationally. ${ }^{9}$ This makes this injury more common than breast cancer, HIV infection/AIDS, spinal cord injury and multiple sclerosis combined. ${ }^{10}$ The Public Health Agency of Canada reported that TBI is among the most costly neurological conditions, set to cost 8 billion dollars by 2031, not taking into account personal repercussions in terms of injured men's and women's struggles to perform familiar and societal roles and responsibilities. ${ }^{11}$

It has been long known that sex and gender play a major role in the incidence, clinical presentation, manifestation and outcomes of TBI. ${ }^{712-14}$ For example, sex has been implicated in the biological and physiological aspects of brain injury, and the idea of oestrogen as a neuroprotective factor, moderating the impact of brain injury in women, has been extensively explored. ${ }^{12}$ Gender is implicated as a social determinant of outcomes. ${ }^{7}$ For example, women occupied in traditionally feminine sectors have been shown to have more positive experiences with the postinjury return-to-work process relative to their counterparts working in more traditionally masculine environments. ${ }^{15}$ These results, however, are not consistent-it has also been reported that women are more likely than men to reduce their work hours and to stop working altogether after their injury. ${ }^{16} 17$ These variations reflect the novelty of this field, as standardised approaches to sex-specific and gender-specific research are still evolving.

The Acquired Brain Injury and Society Research Laboratory at the Toronto Rehabilitation Institute-University Health Network (UHN) ${ }^{18}$ the largest rehabilitation teaching hospital in Canada, has initiated action on several fronts of rehabilitation research by incorporating sex/gender in all of their research hypotheses, recognising the biological (sex) differences between men and women, and acknowledging the complex and dynamic relationship that exists between sex and the socially determined factors of gender. Results from completed research projects affirm that the same injury (ie, TBI) is expressed and managed differently in men and women. ${ }^{7}$ This inequity is shaped partially by socially created attributions and expectations assigned on the basis of biological sex, and by the behaviours that men and women endorse to be compatible with these socially created standards. It is the intersection of sex and gender (ie, sex/gender) that has been shown to be responsible for major variations in the risk of exposure to injury with a TBI outcome; the load of comorbid disorders at the time of injury and at follow-up; help-seeking behaviours; post-traumatic symptom load and severity; access to, control, and use of healthcare resources; response to rehabilitation interventions; and most importantly, lifetime outcomes that are of relevance to patients, their significant others (SOs) and clinicians. ${ }^{7}$

With recognition of the need for sex/gender-sensitive knowledge translation (KT) interventions in TBI clinical practice guided by research evidence, a novel multistep integrated research programme is being created with the development, application and evaluation of an evidencebased sex-specific and gender-specific educational KT intervention. The aim of this research programme, funded by the Canadian Institutes of Health Research (CIHR), under the team grant titled 'A Gender-Transformative Approach to Improve Outcomes and Equity among Persons with Traumatic Brain Injury' (\#CGW126580), is to advance a sex/gender agenda in clinical policy and practice. As the team completes its first 3-year funding period (2017-2020), a study protocol is being outlined for a programme of research that seeks to (1) apply the existing evidence base, the knowledge users' understanding, attitudes and needs, and experts' feedback on how best to support sex/gender-sensitive educational interventions in TBI; (2) develop and test patient and SO-targeted, and clinician-targeted intervention programmes, focused on developing a greater understanding of the gender and its impact in persons with TBI, and on evaluation/assessment of gender and gendered roles, and psychosocial profiles associated with TBI and (3) research whether application of these educational materials is effective (ie, changes personal and professional knowledge and attitudes about sex/gender-related vulnerabilities in the context of TBI).

This protocol has been developed by a team with expertise in KT, sex-based and gender-based analysis, brain injury medicine, rehabilitation science, psychology, social theory, safety science and behaviour change (table 1), and has incorporated critical input from the leaders of other CIHR sex-sensitive and gender-sensitive programmes ${ }^{19}$ and an internal ethics advisory group. Publication of this protocol aims to strengthen the scientific rigour of the proposed research programme by creating an early scientific record of the study's methodology.

\section{METHODS}

\section{Ethics}

Informed consent was modelled after a typical consent form used for clinical trials. In order to develop and standardise items to measure sex-specific and gender-specific outcomes relevant to TBI, the Patient-Reported Outcomes Measurement Information System will be used. ${ }^{20}$

\section{Patient and public involvement}

Building infrastructure to facilitate the implementation of sex/gender-sensitive research findings into clinical considerations in TBI requires conceptualisation, development, adoption and integration of new ideas and practices. Given that a consensus-based framework for sex-specific and gender-specific outcomes in TBI has not been developed, a hypothesised framework was formed 
Table 1 List of team members and affiliations and partners organisations

\section{Research programme team}

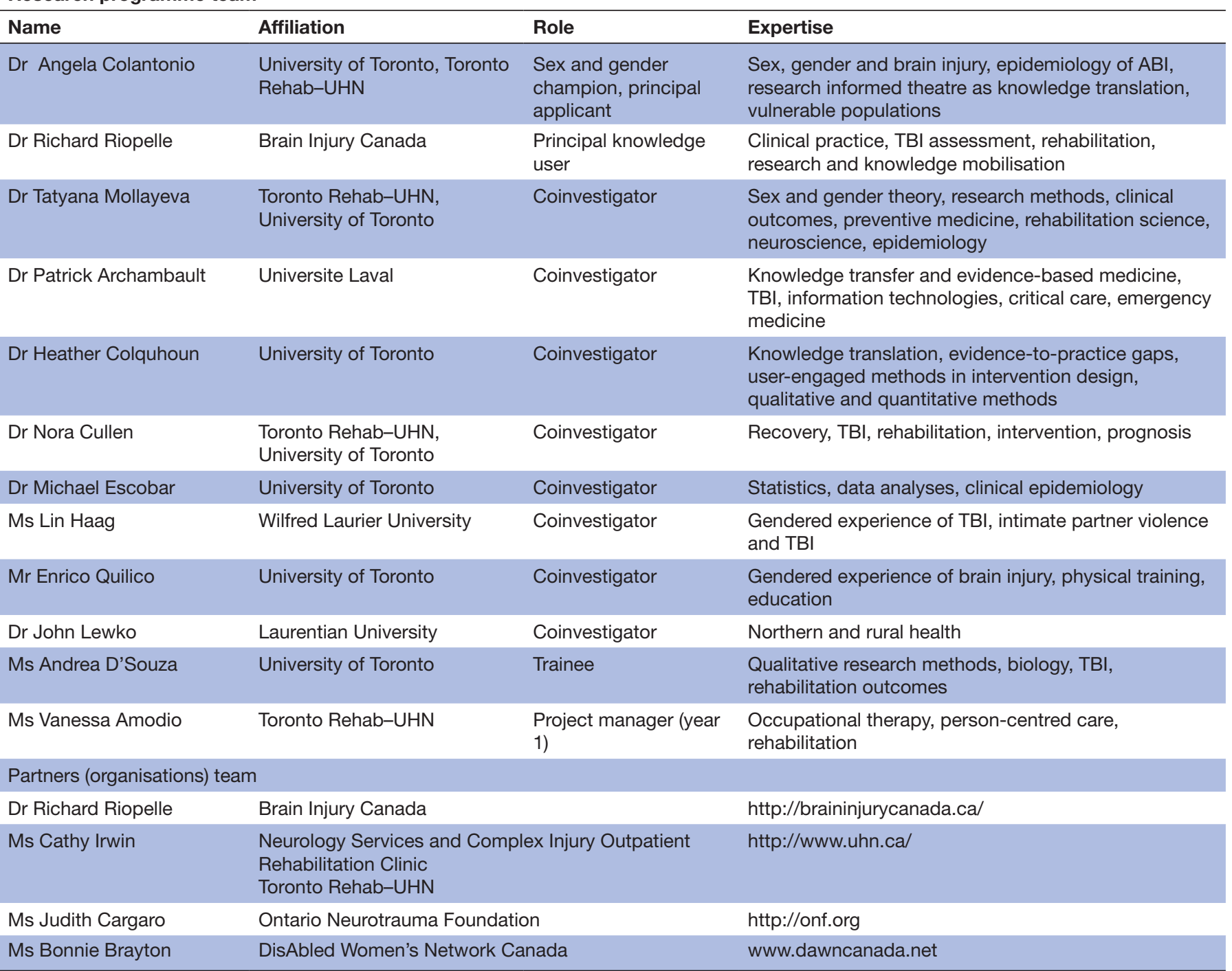

$\mathrm{ABI}$, acquired brain injury; TBI, traumatic brain injury; UHN, University Health Network.

and will be further explored with qualitative and quantitative methodologies (figure 1). This research programme follows the standards of patient and public involvement in research, ${ }^{21}$ enabling decision-making to be shared more equitably with patients and the public (through our partner organisations, table 1) along a continuum of all phases of the programme, including consultations with partnership organisations, active involvement in the research process and shared leadership.

To enhance user experience and engagement, the educational material will be developed with the direct involvement of the knowledge users (ie, men and women of various age groups and psychosocial profiles, their SOs and the clinicians attending to patients with TBI) to inform on educational product features and design preferences. For the purposes of this work, the following definition for SOs was used: 'A person who is important to one's well-being'. ${ }^{22}$ Using a knowledge-user feedback framework, ${ }^{23}$ current scientific evidence and the research team's expertise, all concepts and ideas will be documented and incorporated in the educational material. It is expected that persons with lived experience, their SOs and clinicians working in the TBI context will present unique experiences, values and preferences, and therefore, it is anticipated that the programme developed will be applicable to all knowledge users. Further collaboration with art product developers will aid in operationalising person-specific educational components specifically designed to appeal to the respective knowledge users. Finally, healthcare professionals involved in this project, as well as partner organisations, have actively embraced involvement and collaboration in the research programme and have actively participated in monthly team meetings, influencing the process.

\section{RESEARCH OBJECTIVE 1A: INTERVIEW OF KNOWLEDGE USERS}

The initial stage of the research programme involves documentation of personal experiences of persons with TBI, 


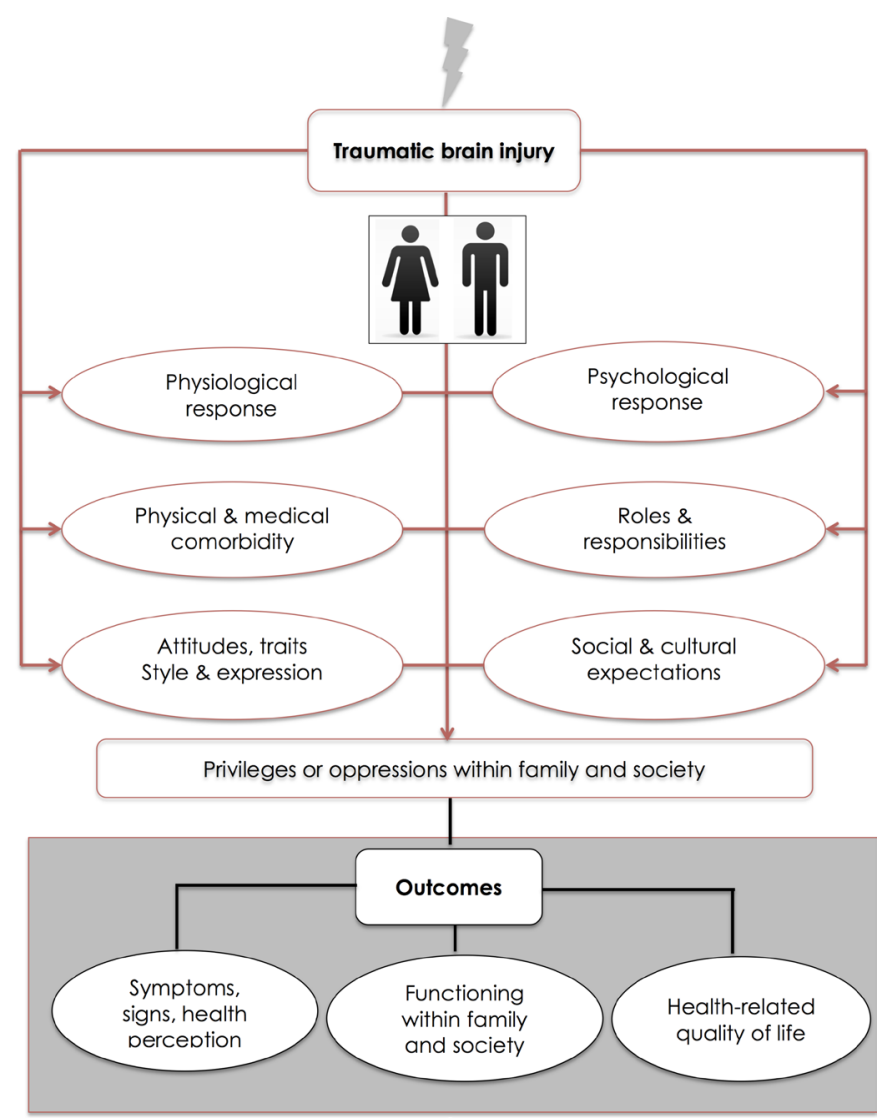

Figure 1 Inter-related construct of sex and gender in traumatic brain injury; will be referred to as sex/gender. Unidirectional arrows between constructs (ie, rectangles) and items (ie, ovals) represent reflective models.

their SOs and clinicians involved in their care. Because of the lack of insight and theories to explain sex/gender influences in TBI, this research aims to summarise the main structural components of the sex/gender in TBI theory in order to support educational interventions in TBI, an injury that frequently affects socially marginalised populations. ${ }^{7}$ However, a comprehensive theory of sex/gender influences in TBI, which requires a complete range of personal circumstances, cultural and socioeconomic conditions and mechanisms of injury, severity, and stages of TBI across the continuum of care, is not the focus of the research programme.

\section{Qualitative interviews}

Purposive recruitment will be facilitated through the UHN and will include persons with acute TBI, persons with chronic TBI from the community entering rehabilitation programmes and their SOs, and treatment providers from these settings. Our selection of participants involves a purposive (or theoretical) sampling strategy, which offers a degree of control for selection bias present within groups. $^{2425}$ It is estimated that at least five men and five women with mild TBI (concussion) and the same with moderate-severe TBI from rehabilitation setting at the acute and chronic stages after their injuries are needed for interview; two SOs per injury severity group per stage of injury; and two men and two women clinicians dealing with patients at the acure and chronic post injury phases.

The total sample size would therefore be at least 40 persons with TBIs of various severities in the two post injury stages (10 participants $\times 2$ severity groups $\times 2$ stages after the injury), eight SOs $(2 \times 2 \times 2)$ and 16 clinicians attending to persons with TBI $(4 \times 2 \times 2)$. The proposed sample size is consistent with requirements for qualitative sampling in a relatively homogeneous group of patients with TBI and should be sufficient to facilitate theoretical saturation. $^{26}$

\section{Participants}

Participants will be screened for eligibility according to the following criteria:

- Inclusion criteria: patients with a primary diagnosis of TBI and their SOs, and clinicians working with patients with TBI.

- Exclusion criteria: neurocognitive problems or limited literacy resulting in an inability to complete an interview/questionnaire with or without help of research personnel or an SO.

\section{Additional information on patient participants}

Adult ( $\geq 18$ years) Ontario residents who entered the UHN facilities with a diagnosis of TBI as the most responsible diagnosis in the referral field will be considered for inclusion in this study. TBI is generally defined as a structural and/or physiological disruption of brain function as a result of an external force. ${ }^{8}$

Clinical team members at therecruitment site will make patients and SOs aware of the ongoing project during orientation meetings. The central recruiter at the UHN will screen all patients' clinical files to ensure eligibility for participation (eg, primary diagnosis of TBI and cognitive and linguistic ability to participate in research) and will contact eligible participants and provide information about the study purpose, what is involved, risks and benefits, confidentiality, and right to withdraw, verbatim and in written form. In cases of cognitively challenged patients, as recorded in clinical files, acceptable representatives will be contacted by the centralised recruiter: if the potential participant will regain capability to participate in research during the course of the study, they will be able to re-consent or decline further participation.

The following are examples of lines of questioning to be used in the interviews:

- What does being a woman/man living with TBI mean to you-how do you define and describe this?

- What does it mean to be 'feminine' / masculine'?

For clinicians, the following will be asked as part of the first question:

- What do you know about sex and gender differences in TBI with regard to pathophysiology, risk factors, clinical manifestations and overall outcomes?

- What are some good ways to deliver educational interventions on the topic of sex and gender influences in TBI? 
Refer to online supplementary file 1 for the complete set of questions featured in the interview.

\section{Data analysis}

Each participant interview (in person or by phone) will be audio-recorded, transcribed and de-identified for the protection of the participants. One researcher will complete the transcription following each interview, and a minimum of two researchers will systematically analyse each transcribed interview shortly thereafter, pursuing themes as they arise. Transcribed data from interviews will be developed into a conceptual model based on grounded theory principles. ${ }^{2728}$

This method of data analysis involves three phases: (1) open coding, which is the process of chunking data into smaller units, examining, comparing, conceptualising and placing units into related categories; (2) axial coding, which involves reassembling data into categories on the basis of relations identified in the data; and (3) selective coding, involving the process of development of the central theme found in the data. ${ }^{28}$ The validity of the analysis will be strengthened through the use of two or more data analysers per transcription and regular team meetings to discuss the data and analyses. The purpose of using multiple coders and team discussions is to verify the accuracy of each other's codes, to ensure that the results were appropriately derived from the study data, thus improving scientific rigour. ${ }^{27}$

\section{RESEARCH OBJECTIVE 1B: EVIDENCE SYNTHESIS ON THE TOPIC OF INTEREST}

While the responses of patients and their SOs and clinicians will inform the work, the development of educational materials cannot proceed without collection and synthesis of the existing evidence. The assumption of evidence-based practice is that there are things that the working team must know in order to conduct practice professionally. Therefore, during the initial phase of the project, a systematic review of the available research applying sex and gender analysis in the TBI context will be conducted (review currently in progress). ${ }^{29}$ The review will be conducted and reported in accordance with the Preferred Reporting Items for Systematic Reviews and Meta-Analyses guidelines. ${ }^{30}$ All peer-reviewed English language publications in adults with TBI from inception to February 2018 found through Cochrane Central Register of Controlled Trials (CENTRAL), CINAHL, Embase, Ovid MEDLINE(R) (including epub ahead of print, in-process and other non-indexed citations, and Ovid MEDLINE(R) Daily), PsycINFO and Web of Science were systematically searched. Search strategies included the use of textwords and subject headings (eg, $\mathrm{MeSH}$ and Emtree) related to constructs of (1) TBI and (2) sex or (3) gender. Quality will be evaluated using published guidelines. Research questions for this systematic review concern understanding differences between men and women with TBI across a continuum of care and lifespan in (1) functioning within family and society before and after the injury; (2) health-related quality of life; (3) social and cultural expectations; (4) roles and responsibilities; (5) physical and mental health comorbidity; and (6) attitudes, traits and expressions (figure 1). Results will be grouped by relevance to gender assessment (how to gather and interpret findings from medical history, physical examination and care/SO research), aetiology of sex and gender dimorphism, prognosis (how to gauge the person's likely clinical course of TBI in a social context, as well feasibility and appropriateness of sex-based and gender-based analysis within the different social contexts in which the research takes place). The detected results will be exemplified within the developed educational material.

\section{RESEARCH OBJECTIVE 2: DEVELOPMENT OF EVIDENCE-BASED KNOWLEDGE INTERVENTION}

The main aims and anticipated deliverables for research objective 2 are to develop (1) a patient and SO-directed intervention entitled 'Gender Implications for Canadians Living with TBI (GIC-TBI-P)' focused on constructing and understanding sex and gender roles on a TBI background; and (2) a professional intervention (passive or active, to be determined in research objective 1) entitled 'Gender Implications for Clinicians Attending to Patients with TBI' (GIC-TBI-C) focused on evaluation and assessment of potential sex-related and gender-related strengths and vulnerabilities in the TBI context. Figure 2 presents the steps involved in the development of the educational material, along with the formal strategies for doing so.

\section{RESEARCH OBJECTIVE 3: PARTICIPANT RANDOMISATION AND EFFICACY TRIAL}

Researchers, a man and a woman, trained in the delivery of the GIC-TBI-P and GIC-TBI-C, through completion of an initial training session and refresher training session over a period of 3 months, will be delivering the educational material to knowledge users.

The main aim of research objective 3 (educational intervention and cluster-randomised trial in KT) is to understand the feasibility and effectiveness of educational interventions on knowledge and value judgements concerning sex-related and gender-related strengths and vulnerabilities in knowledge users.

\section{TRIAL METHODS}

\section{Baseline measures and treatment experiment}

Participants from each clinical setting will be randomly selected and assigned to their respective patient-targeted, SO-targeted, and clinician-targeted focus groups for pretesting. Baseline characteristics will be recorded, in addition to the data on knowledge and attitudes with regard to sex and gender in TBI using a questionnaire. Use of the questionnaire is intended to minimise socially 
desirable responses about sensitive topics as respondents may not be prepared to explicitly reveal their knowledge and attitudes.

The baseline questionnaire will include information on the research taking place and ask questions related to

- Demographics (age, culture, religion, education, income, employment, children in the household and living conditions).

- Marital factors (age at and length of marriage).

- Gender roles (cultural values and expectations).

- Family and community roles.

- Sexual risk behaviours.

- Perceived privileges and oppressions within family and society.

- Level of masculinity and femininity.

- Past and current clinical history and health status (including physical and mental health, and status of sleep function).

- Functioning level (community integration and perceived disability).

Refer to online supplementary file 2 for the complete set of questions featured in the questionnaire.

\section{Outcome measures}

The knowledge (\#1) and attitudes (\#2 and \#3) questionnaire will feature questions of the following sort (to be refined as common themes from qualitative interviews and the systematic review emerge):

1. Are the early symptoms of TBI the same for women and men?

- Yes.

- No.

- I am not sure.

If no, how do you think women and men differ?

2. Do you believe that language used by clinicians can either reinforce or minimise gender stereotypes?
For example, a common stereotype is women over-reporting the severity of their post-TBI symptoms.

A stereotype pertaining to men may have to do with their involvement in their injury, for example, due to intoxication, high-risk behaviours.

These stereotypes may or may not be supported by evidence. Answer the above question based on your experiences in the healthcare system and your feeling on whether or not the manner in which clinicians speak to you minimise or reinforce stereotypes related to men and women with TBI.

- Yes, it can reinforce stereotypes.

- Yes, it can minimise stereotypes.

- No, they do neither.

- I am not sure.

If yes, how do you think this is so?

3. Is it necessary to use gender-neutral language to minimise the power imbalance between care provider and care user?

- Yes.

- No.

- I am not sure.

If yes/no, comment on how you think use of gender-neutral language will/will not be of benefit for the patientprovider dynamic:

Refer to online supplementary file 3 for the complete set of questions featured in the questionnaire.

\section{Randomisation procedure}

Participants will be randomly assigned by computer generation to either one of the experimental educational groups, or a control group, which will not take part in any intervention. Final modes of delivery of educational interventions will be determined after completion of the first phase of the project. The following structure and statistical approach described should be treated as preliminary, subject to modification.

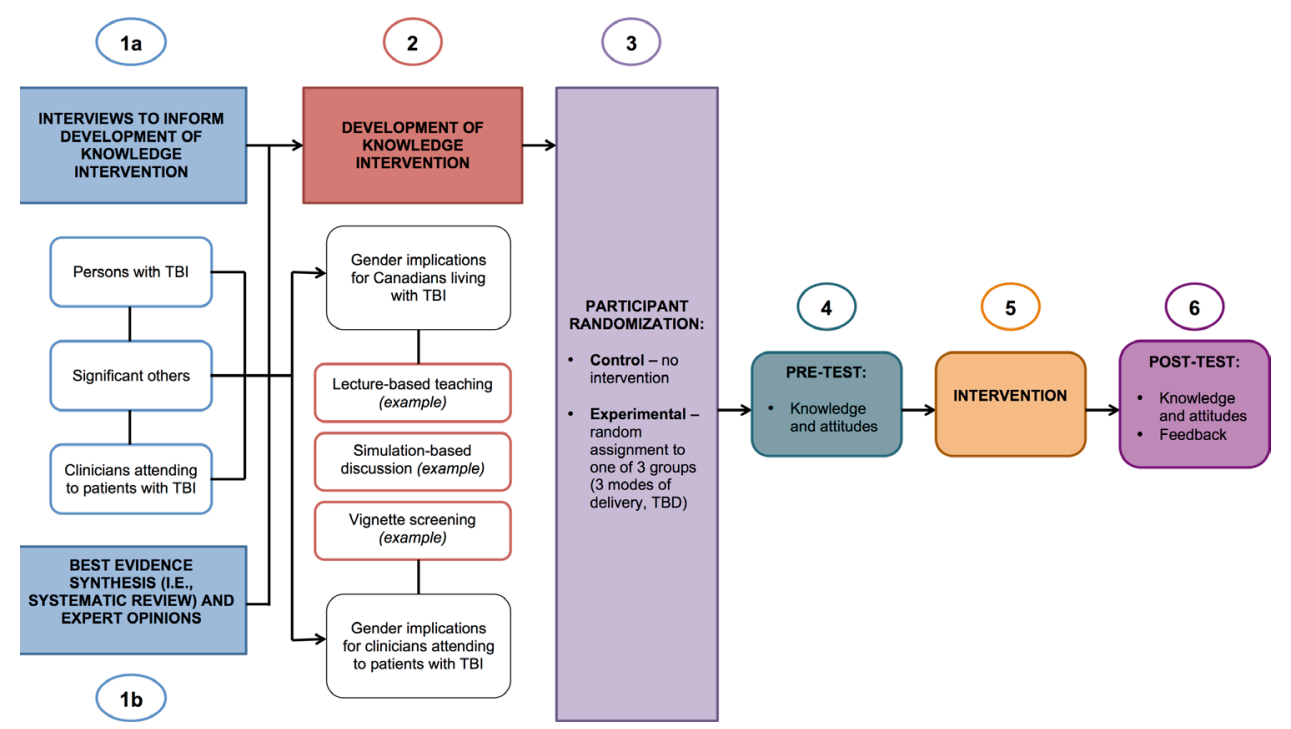

Figure 2 Overview of proposed research programme, commencing with knowledge user interviews and best evidence synthesis to inform development of intervention, followed by randomisation of participants, administration of preintervention questionnaire, intervention exposure and completion of postintervention questionnaire by all participants. TBI, traumatic brain injury. 
Attitudinal data will be collected immediately following delivery of each mode of intervention. In the first experiment (intervention 1), experimental vignettes will be shown once, and further attitudinal data will be collected from participants immediately following screening. In the second experiment (intervention 2), a 1-hour lecture will precede collection of attitudinal data immediately after delivery. For the third experiment (intervention 3), a simulation-based discussion will be offered, following which, attitudinal data will again be collected. The control group (intervention free) will be left untreated, and we will collect follow-up attitudinal data two or more weeks after the baseline assessment. According to the experimental design, the probability of receiving at least one active intervention reaches $75 \%$ (figure 2).

Baseline assessment will be conducted in all groups at potentially different times, one group at a time. Participants in the control group will not be aware that they are assigned to a control group. Two weeks later, participants in the control group will be invited to complete a follow-up assessment only, while other groups will be asked to complete a follow-up assessment following delivery of educational interventions. By doing this, we will ensure the least possible burden is placed on research participants, in terms of daily testing, reasonably equal probability to seek answers to unknown questions and educate themselves, and the ability of research personnel to run the educational programme, taking into account human and material capacity.

\section{Sample size calculation}

Participants will include injured women and men from two clinical settings (acute care and rehabilitation care), recruited with the support of the UHN. The targeted number of recruited participants is 100 (table 2).

\begin{tabular}{|c|c|c|c|c|}
\hline Sampling & Gender & $\begin{array}{l}\text { Acute } \\
\text { stage } \\
\text { after the } \\
\text { injury }\end{array}$ & $\begin{array}{l}\text { Chronic } \\
\text { stage } \\
\text { after the } \\
\text { injury }\end{array}$ & Tota \\
\hline \multirow[t]{2}{*}{ mTBI/SRC } & M & 10 & 10 & 40 \\
\hline & W & 10 & 10 & \\
\hline \multirow{2}{*}{$\begin{array}{l}\text { Moderate-severe } \\
\text { TBI }\end{array}$} & $\mathrm{M}$ & 5 & 5 & 20 \\
\hline & W & 5 & 5 & \\
\hline \multirow[t]{2}{*}{ Significant others } & M & 5 & 5 & 20 \\
\hline & W & 5 & 5 & \\
\hline \multirow{2}{*}{$\begin{array}{l}\text { Clinicians treating } \\
\text { patients at } \\
\text { different stages } \\
\text { after the injury }\end{array}$} & M & 5 & 5 & 20 \\
\hline & W & 5 & 5 & \\
\hline Total & & 50 & 50 & 100 \\
\hline
\end{tabular}

$M$, men; mTBI, mild traumatic brain injury; SRC, sport-related concussion; TBI, traumatic brain injury; W, women.
The sample size is limited to 100 recruited participants due to potential constraints in the ratio of fully eligible, available participants, to the total number of patients interested in the research. Therefore, a realistic estimate of 100 participants $(n=25$ per experimental condition) is achievable and is also supported by a minimum number of cases analysis. A working guideline to identify the minimum number of cases to include in the study by Peduzzi and colleagues was used. ${ }^{31}$ For $\mathrm{k}$ independent variables and $p$ as the smallest of the proportions of negative or positive cases in the population, the minimum number of participants is $10 \times \mathrm{k} / \mathrm{p}$. Our previous studies of the Ontario population found an approximate 60:40 men:women composition when TBIs of all injury severities are taken into account. By taking the minimum proportion value (ie, $40 \%$ women) and setting $\mathrm{k}=2$, the minimum number of cases to be included in the present study is $10 \times 2 / 0.4 \approx 80$. According to this approach, the proposed sample size of 100 is adequate to answer the research question posed and account for attrition.

\section{Data analysis}

The following main hypotheses, with respect to intervention effectiveness, compare each intervention arm to the control arm, as well as the magnitude of change between intervention arms from preintervention to postintervention: (1) there will be greater changes in each intervention arm, when compared with the control arm, for the knowledge and attitudes towards sex and gender considerations in TBI; (2) there will be differences in the magnitude of change between formats of intervention from preintervention to postintervention; and (3) sex and gender knowledge will influence the magnitude of changes observed in the effectiveness of KT interventions. Analyses will be conducted following the Consolidated Standards of Reporting Trials guidelines for randomised trials. ${ }^{32}$

\section{Measure of outcome: knowledge and attitudes}

The primary outcome variable is the absolute change in knowledge from preintervention to postintervention, and the secondary outcome variable is the absolute change in attitudes from preintervention to postintervention, as determined by the answers given to the questionnaire described in the Outcome measures section.

\section{Statistical analysis}

All analyses were performed with SAS V.9.3. Data will be tested for normal distribution with Kolmogorov-Smirnov tests. The groups will be analysed for differences in the baseline age, education, sex and TBI severity using analysis of variance or $\chi^{2}$ tests where appropriate. For all statistical comparisons, the significance level will be set at $\alpha \leq 0.05$.

To test hypothesis 1, a simple two-stage analysis will be used: first, summary measures (ie, means) on knowledge and attitudes will be generated for each intervention arm and the control, preintervention and postintervention; 
second, preintervention and postintervention values will be subtracted to give the mean change in attitude by intervention. A t-test comparing the magnitude of change within each arm compared with the control will be applied.

To test hypothesis 2 , for each intervention arm and the control arm, participant postintervention values will be compared with preintervention values using Cohen's d. ${ }^{33}$ Cohen's d, determined by calculating the mean difference between two groups, and then dividing the result by the pooled SD, will be derived for the whole group in each intervention arm and the control.

The magnitude of change observed in the intervention arm will be compared (hypothesis 3), taking the sex and gender of respondents into account. Tests of significance will be performed for interactions between sex and age, education, occupation, family status, levels of femininity/ masculinity and income level, taken from the baseline questionnaire, to explore the role of gender. In addition, interaction between the magnitude of change and time will be explored. With data satisfying the assumptions for parametric analysis, measures of effect size (hypothesis 3) will be computed using Hedges' g, or corrected effect size. ${ }^{34}$ If the SDs of the effect size are found to be significantly different between intervention arms, we will choose Glass's delta instead of Hedges' g. ${ }^{34}$

\section{Ethics and dissemination}

Successful implementation of sex and gender in research and practice requires that policymakers, patients and clinicians have sufficient understanding of the sex/ gender implications in TBI. However, as with many social factors, topics of gender and sex, specifically as they relate to health and medicine (ie, to outcomes following TBI), are unfamiliar to many patients, SOs and clinicians. Therefore, evidence-based educational interventions are needed. Development of educational interventions to support brain injury policy and practice is proposed, specifically KT interventions suited to those who will use them (ie, patients with TBI, their SOs and clinicians), using a non-discriminatory approach to sex and gender in TBI. As such, the evidence-based KT material will be developed and delivered, taking into account the biological and social circumstances of women and men, and evaluating structural inequalities. Key issues to be explored include those that affect experiences post TBI, such as mechanism of injury (ie, assault, fall from elevation and exposure to explosion); parenting and caring roles; poverty and isolation; feelings of safety at home; and meaningful professional, familial, and societal roles, among others. A shift is anticipated towards KT activities that are personal and sensitive to individual needs and in which the greatest ownership of evidence is to be given to those who are best placed to make changes (ie, patients with TBI, their SOs and clinicians).

The focus on the importance of patients', SOs' and clinicians' training should be especially recognised, involving a multifaceted approach geared at increasing awareness of key gender issues directly influencing patient's care and offered services, sex-specific and gender-specific inequalities, personal attitudes, as well as structured diversity of experiences among and between women and men.

Dissemination of research is incomplete without engaging knowledge users in efforts to translate results to practice. Collaboration with partner organisations (table 1) can enhance knowledge dissemination. Publishing the results of the systematic review and the qualitative interviews allows peer-review feedback and citations of these works in the educational intervention study paper. Educational material development steps and KT intervention results will be disseminated through presentations and publications, and are expected to facilitate early implementation by researchers and educators. All KT interventions will be developed with the aim of integration into training of patients, SOs and clinicians.

There are forthcoming challenges to the programme. A limitation for the inclusion of a multistep research programme, including knowledge synthesis and qualitative interviews, is a greater commitment of time and effort from researchers and knowledge users early in the study. However, it is anticipated that these early efforts will capture sex and gender effects in TBI, especially through the unique voices and narratives of women and men with TBI, their SOs and clinicians. Their active involvement in the development of their own educational interventions is expected to play a facilitative role in helping all parties take into account the structural diversity of experiences of women and men with TBI, within the broader societal equality and diversity paradigm. Although the programme is comprehensive and contains many of the key ingredients for success (knowledge synthesis, qualitative and quantitative research methodologies, knowledge user's and community partner's feedback), there remains a risk that these findings may lack credibility in the eyes of those who value multisite research programmes.

\section{CONCLUSIONS}

Developed and implemented through knowledge synthesis and collaborative discussion with academic colleagues, clinical partners and knowledge users, the proposed programme, "A Gender Transformative Approach to Improve Outcomes and Equity among Persons with Traumatic Brain Injury', aims to advance gender strategies in TBI practice and care and to support development of gender-specific research and clinical guidelines for TBI. It is also a good step towards the eventual realisation of the need for sex-customised and gender-customised interventions, both preventive and therapeutic.

Collaborators Richard Riopelle; Patrick Archambault; Nora Cullen; Michael Escobar; John Lewko; Cathy Irwin; Judith Gargaro; Bonnie Brayton.

Contributors TM: study conception and design, definition of programme steps, protocol development and questionnaire development, ethics board applications and drafting of the manuscript. AC: study conception and design, research programme supervision and implementation; supervision of ethics board 
applications; and critical revision of the study protocol. HC, EQ and HH: significant contributions to study conception and design, and protocol development and refinement. SM: literature search and protocol development; and questionnaire development, drafting of the manuscript and all visual presentation featured in this work. VA: ethics board modifications and annual renewal, study piloting and data collection (phase 1 of the programme). AD: literature search and data analysis. All authors critically reviewed the final version of the manuscript and gave the final approval of the submitted manuscript.

Funding This research program is supported by the Canadian Institutes of Health Research (CIHR) Institutes of Gender and Health grant \#CGW-126580. The study was peer-reviewed and funded by the CIHR Grant-Institute for Gender and Health (\#CGW-126580). Ethics approval was granted by the Toronto Rehabilitation Institute-University Health Network and the University of Toronto Ethics Boards. During the work on this manuscript, the first author was supported by the postdoctoral research grant from the Alzheimer's Association (AARF-16-442937). The funders had no role in the study design, decision to publish or preparation of the manuscript. This research program was developed with endorsement by patient and public organizations (table 1). The critical need to address sex and gender in brain injury research and rehabilitation, to ensure appropriate, equitable interventions for all patients, was highly endorsed by the leadership of these organizations, which reviewed the research program prior its submission to the funding agency (CIHR). These organizations acted as a liaison between our research team, the patients and the public. Dr Richard Riopelle, a member of the Brain Injury Canada Board of Directors and a primary knowledge user on the grant, and all our other partners are committed to engaging in further knowledge transfer events, meetings and dialogues to expand, share and disseminate project results and educational tools.

Competing interests None declared.

Patient consent for publication Not required.

Ethics approval The study protocol was approved by the ethicscommittees at the clinical (Toronto Rehabilitation Institute-UniversityHealth Network, CAPCR: 17-5663) and academic research (University ofToronto, Protocol \# 000361174) institutions at which the study is taking place.

Provenance and peer review Not commissioned; externally peer reviewed.

Open access This is an open access article distributed in accordance with the Creative Commons Attribution Non Commercial (CC BY-NC 4.0) license, which permits others to distribute, remix, adapt, build upon this work non-commercially, and license their derivative works on different terms, provided the original work is properly cited, appropriate credit is given, any changes made indicated, and the use is non-commercial. See: http://creativecommons.org/licenses/by-nc/4.0/.

\section{REFERENCES}

1. Canadian Institutes of Health Research. Definitions of Sex and Gender. 2015 http://www.cihr-irsc.gc.ca/e/47830.html (Retrieved 30 Mar 2018).

2. National Institutes of Health. NOT-OD-15-102: consideration of sex as a biological variable in $\mathrm{NIH}$-funded Research. https://grants.nih. gov/grants/guide/notice-files/NOT-OD-15-102.html (Retrieved 30 Mar 2018).

3. Polderman TJC, Kreukels BPC, Irwig MS, et al. The Biological Contributions to Gender Identity and Gender Diversity: Bringing Data to the Table. Behav Genet 2018;48:95-108.

4. Tannenbaum C, Greaves L, Graham ID. Why sex and gender matter in implementation research. BMC Med Res Methodol 2016;16:145.

5. WHO. Gender, equity and human rights. https://www.who.int/ gender-equity-rights/understanding/gender-definition/en/ (Retrieved 16 Dec 2018).

6. Colantonio A. Sex, gender, and traumatic brain injury: a commentary. Arch Phys Med Rehabil 2016;97(2 Suppl):S1-4.

7. Mollayeva T, Mollayeva S, Colantonio A. Traumatic brain injury: sex, gender and intersecting vulnerabilities. Nat Rev Neurol 2018;14:711-22.

8. Brain Injury Association of America. About Brain Injury. http://www. biausa.org/about-brain-injury.htm (Retrieved 30 Mar 2018).
9. Quaglio G, Gallucci M, Brand H, et al. Traumatic brain injury: a priority for public health policy. Lancet Neurol 2017;16:951-2.

10. Center for Disease Control and Prevention. Brain Injury Basics. https://www.cdc.gov/headsup/basics/index.html (Retrieved 16 Dec 2018).

11. Brain Injury Association of Canada. National population health study of neurological conditions: Projections of the impact of hospitalized traumatic brain injury on the Canadian economy, 2011 to 2031. http://braininjurycanada.ca/wp-content/uploads/CONF2014_Dr-RonWall-Indirect-Costs-of-TBI-BIAC-September-2014.pdf (Retrieved 16 Dec 2018).

12. Emerson CS, Headrick JP, Vink R. Estrogen improves biochemical and neurologic outcome following traumatic brain injury in male rats, but not in females. Brain Res 1993;608:95-100.

13. Jullienne A, Salehi A, Affeldt B, et al. Male and female mice exhibit divergent responses of the cortical vasculature to traumatic brain injury. J Neurotrauma 2018;35:1646-58.

14. Biswas RK, Kabir E, King R. Effect of sex and age on traumatic brain injury: a geographical comparative study. Arch Public Health 2017;75:43.

15. Stergiou-Kita M, Mansfield E, Sokoloff S, et al. Gender influences on return to work after mild traumatic brain injury. Arch Phys Med Rehabil 2016;97(2 Suppl):S40-5.

16. Corrigan JD, Lineberry LA, Komaroff E, et al. Employment after traumatic brain injury: differences between men and women. Arch Phys Med Rehabil 2007;88:1400-9.

17. Bounds TA, Schopp L, Johnstone B, et al. Gender differences in a sample of vocational rehabilitation clients with TBI. NeuroRehabilitation 2003;18:189-96.

18. Research at UHN. http://www.uhnresearch.ca/institutes/tri (Retrieved 16 Apr 2018)

19. Canadian Institute of Healthcare Research. Impact of Gender on Knowledge Translation Interventions. http://www.cihr.gc.ca/e/50471. html (Retrieved 30 Mar 2018)

20. Cella D, Yount S, Rothrock N, et al. The Patient-Reported Outcomes Measurement Information System (PROMIS): progress of an $\mathrm{NIH}$ Roadmap cooperative group during its first two years. Med Care 2007;45(5 Suppl 1):S3-11.

21. Ocloo J, Matthews R. From tokenism to empowerment: progressing patient and public involvement in healthcare improvement. BMJ Qual Saf 2016;25:626.32.

22. Merriam-Webster. Definition of significant other. (Retrieved $16 \mathrm{pr}$ 2018).

23. Bartholomew LK, Parcel GS, Kok G, et al. Intervention mapping: designing theory and evidence-based health promotion programs: Mountain View: Mayfield Publishing Company, 2001

24. Kuzel AJ. Sampling in qualitative inquiry. In: Crabtree BF, Miller WL, eds. Doing qualitative research. London: Sage, 1992:31-44.

25. Mays N, Pope C. Rigour and qualitative research. BMJ 1995;311:109-12.

26. Tran VT, Porcher R, Tran VC, et al. Predicting data saturation in qualitative surveys with mathematical models from ecological research. J Clin Epidemiol 2017;82:30543-1.

27. Barbour RS. Checklists for improving rigour in qualitative research: a case of the tail wagging the dog? BMJ 2001;322:1115-7.

28. Strauss A, Corbin J. Basics of qualitative research: grounded theory procedures and technique4s. Newbury Park: Sage Publications, 1990.

29. Mollayeva T, Pacheco N, Mollayeva S, et al. Sex, gender, and intersecting vulnerabilities in traumatic brain injury. PROSPERO. 2018 http://www.crd.york.ac.uk/PROSPERO/display_record.php?ID= CRD42018098697 (Retrieved 19 Jun 2018).

30. Moher D, Liberati A, Tetzlaff J, et al. Preferred reporting items for systematic reviews and meta-analyses: the PRISMA statement. PLoS Med 2009;6:e1000097.

31. Peduzzi P, Concato J, Kemper E, et al. A simulation study of the number of events per variable in logistic regression analysis. $J$ Clin Epidemiol 1996;49:1373-9.

32. CONsolidated Standards of Reporting Trials. $2010 \mathrm{http}: / / \mathrm{www}$. consort-statement.org/consort-2010 (Retrieved 16 Dec 2018).

33. Wassertheil S, Cohen J. Statistical power analysis for the behavioral sciences. Psychol Methods 2003;8:254-74

34. Hedges LV. Distribution theory for glass's estimator of effect size and related estimators. Journal of Educational Statistics 1981;6:107-28. 